\title{
Psychosis reactivity to cannabis use in daily life: an experience sampling study
}

Cécile Henquet, Jim van Os, Rebecca Kuepper, Philippe Delespaul, Maurice Smits, Joost à Campo and Inez Myin-Germeys

\section{Background}

Little is known about the experiential dynamics of the interaction between cannabis and vulnerability to psychosis.

\section{Aims}

To examine the effects of cannabis on psychotic symptoms and mood in patients with psychosis and healthy controls.

\section{Method}

Patients with a psychotic disorder $(n=42)$ and healthy controls $(n=38)$ were followed in their daily lives using a structured time-sampling technique.

\section{Results}

Daily life cannabis use predicted subsequent increases in positive affect and in patients, but not in controls, decreases in negative affect. In patients, but not in controls, cannabis use predicted increased levels of hallucinatory experiences. Mood-enhancing properties of cannabis were acute, whereas psychosis-inducing effects were sub-acute. There was no direct evidence for self-medication effects in daily life.

\section{Conclusions}

Patients with psychosis are more sensitive to both the psychosis-inducing and mood-enhancing effects of cannabis. The temporal dissociation between acute rewarding effects and sub-acute toxic influences may be instrumental in explaining the vicious circle of deleterious use in these patients.

\section{Declaration of interest}

None.
Cannabis use is associated with an increased risk of psychotic symptoms in individuals from the general population. ${ }^{1}$ In patients with an established psychotic disorder, cannabis has a negative impact on illness course, as evidenced by more and earlier relapses, more frequent hospitalisations, and poorer psychosocial functioning. ${ }^{2-4}$ Other epidemiological work, however, has suggested that cannabis may reduce negative and affective symptoms in patients with schizophrenia. ${ }^{5}$ Studies using selfreport questionnaires to investigate motives that maintain cannabis use among individuals with psychotic disorders indicate that the principal motives for use in this group are enhancement of positive affect, social acceptance and coping with negative affect. ${ }^{6}$ Additional evidence that patients may use cannabis to self-medicate distress was reported in a population-based study where vulnerability for psychosis (measured by means of a questionnaire) predicted future cannabis use in those who had never used cannabis before the onset of psychotic symptoms. Two earlier population-based studies, however, had found no evidence for such reverse causality. ${ }^{8,9}$ In order to design successful interventions, epidemiological designs may not be sufficient to provide full insight into the complicated dynamics of cannabis use and its varied effects in patients with psychosis. The current study was therefore designed to further examine, in the context of these complicated dynamics, the association between cannabis use and psychosis using a momentary assessment method. The aims of the current study were to investigate whether:

(a) frequency of cannabis use in daily life fluctuates as a function of mood and psychotic symptom level (i.e. self-medication effects);

(b) cannabis use is associated with subsequent changes in mood and psychotic symptom level;

(c) patients with a psychotic disorder differ from healthy controls in their sensitivity to the psychosis-inducing effects of cannabis; and (d) temporal dynamics of cannabis-induced symptoms are apparent (i.e. short- $v$. long-lasting effects of cannabis can be discerned).

\section{Method}

\section{Participants}

The study sample consisted of 48 patients with a clinical diagnosis of a psychotic disorder and 47 healthy controls. The patients and controls were all frequent cannabis users (current use of at least three times per week). Patients were recruited through in-patient and out-patient mental health service facilities in South Limburg, The Netherlands, and controls were recruited from local 'coffee shops' (cafés where cannabis is sold and consumed legally). Participants were provided with a complete description of the study and written informed consent was obtained. The study was carried out in accordance with the World Medical Association's (WMA's) Declaration of Helsinki on Ethical Principles for Medical Research Involving Human Subjects, as adopted by the 52nd WMA General Assembly, Edinburgh, October 2000. The study was approved by the standing medical ethics committee of Maastricht University Medical Centre. Interview data were used to complete the Operational Criteria Checklist for Psychotic Illness (OPCRIT), ${ }^{10}$ yielding diagnoses according to Research Diagnostic Criteria (RDC) ${ }^{11}$ through the OPCRIT computer program for Windows. In addition, current symptomatology was assessed with the Positive and Negative Syndrome Scale (PANSS) ${ }^{12}$ and additional information with regard to current psychiatric illness, past and current substance use, and other demographic information was collected. Exclusion criteria were: respiratory, cardiovascular or neurological disease and alcohol use in excess of 5 units per day. Pregnant women were also excluded. A personal or family history of psychosis or use of 
antipsychotic medication was a further exclusion criterion for the control group.

\section{Experience sampling method}

The experience sampling method (ESM) is a pseudo-random time sampling self-assessment technique. Previous applications of ESM have demonstrated feasibility, validity and reliability in patients with schizophrenia. ${ }^{13,14}$ Participants received a digital wristwatch, and a paper-and-pen ESM booklet. Twelve times a day on six consecutive days, the watch emitted a beep at random moment in each 85 -minute time block between $07.30 \mathrm{~h}$ and $00.30 \mathrm{~h}$. After each beep, participants were asked to fill in a self-assessment form, collecting reports of affect, thoughts, severity of symptoms and activity rated on 7-point Likert scales at the moment of the beep. Participants were instructed to complete the form immediately after the beep to minimise memory distortion and to record the time at which they completed their report. During a briefing session, the ESM procedure was explained and a practice booklet was completed and discussed with the participant to confirm that the scale format was accurately understood. During the ESM week, participants were contacted by phone in order to ensure that they complied with the instructions. Participants were requested not to use illicit drugs other than cannabis during the 6 consecutive study days; however, no sanctions were involved. None of the participants was a regular user of drugs other than cannabis. During the telephone contacts, participants were asked about their use of other drugs and one person admitted to having used cocaine on the third day of the ESM week. Reports following this cocaine use were excluded from the analyses. At each beep when forms were completed, participants were asked to report the exact time. Reports completed more than 5 mins before and 15 mins after the beep were excluded from the analyses. This was done because previous research has shown that remote answers are less reliable and less valid than reports at the exact moment of the beep. ${ }^{15}$ Participants with less than 24 valid reports were excluded from the analyses, as previous work has shown that measures of individuals with less than $30 \%$ of completed reports are less reliable. ${ }^{15}$

\section{Measures}

Measures regarding cannabis use, mood, and psychotic symptoms were derived from the ESM reports as described below.

\section{Assessment of cannabis use}

Cannabis use, reported after each beep, referred to the period between the previous beep and the current beep (cannabis use, a binary variable). Similarly, cannabis use previous referred to cannabis use during the period between the previous beep and the beep before that. Alcohol use, reported after each beep, referred to the period between the previous beep and the current beep (alcohol use, a binary variable).

\section{Assessment of mood}

Mood states were assessed with 11 mood adjectives rated on a 7 -point Likert scale $(1=$ not at all, $7=$ very) reported after each beep, referring to the mood state at that moment (e.g. 'at this moment I feel anxious'). In previous ESM studies ${ }^{13,14}$ with similar populations (patients with psychosis and healthy controls), a positive and a negative mood scale were identified with factor analysis on the raw within-participant scores of the mood items. For the current analyses, therefore, the mean of the adjectives 'cheerful', 'relaxed', 'happy', 'satisfied', 'enthusiastic', 'overall good' formed the positive affect scale (a continuous variable, Cronbach's $\alpha=0.89$ ), and the mean of 'insecure', 'lonely', 'anxious', 'blue', 'guilty' formed the negative affect scale (a continuous variable, Cronbach's $\alpha=0.80$ ). Positive affect previous

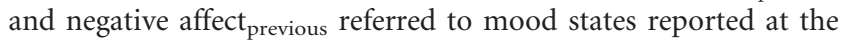
previous beep.

\section{Assessment of psychosis}

Positive psychotic symptoms were assessed with seven items rated on a 7 -point Likert scale $(1=$ not at all, $7=$ very $)$ reported after each beep, referring to the psychotic experiences at that moment. In order to allow self-reporting of psychotic experiences, the items assessing delusional ideation include aspects that can be associated directly with concrete positive psychotic experiences, rather than interpretations of these experiences (e.g. 'at the moment my thoughts are being controlled by others'). Guided by previous studies ${ }^{16,17}$ the mean of 'preoccupied thoughts', 'racing thoughts', 'difficulty expressing thoughts', 'thoughts controlled by others' and 'suspicious' formed the delusions scale (a continuous variable, Cronbach's $\alpha=0.72$ ). Hallucinatory experiences were assessed directly (e.g. 'at the moment I'm hearing voices') as it has been shown in previous studies that patients can distinguish between hearing real voices and verbal hallucinations. ${ }^{18}$ The mean of 'seeing things' and 'hearing things' formed the hallucinations scale (a continuous variable, Cronbach's $\alpha=0.70$ ). Delusions previous $_{\text {and }}$ and hallucinations $s_{\text {previous }}$ referred to psychotic symptom severity reported at the previous beep.

\section{Statistical analyses}

As ESM data have a hierarchical structure with multiple reports (level 1) nested within participants (level 2), multilevel random regression analyses were conducted to account for the variability associated with the two different levels. The odds ratios (for dichotomous variables) and betas (for continuous variables) of these regression analyses are the associations between the independent and dependent variables in the multilevel model. The STATA (version 10 for Windows) multilevel regression XTGEE routine was used for dichotomous variables, and the XTREG routine for continuous variables.

\section{Overall symptom levels}

Differences between patients and controls in overall symptom level were investigated using group $(0=$ controls; $1=$ patients $)$ as independent and negative affect, positive affect, delusions and hallucinations as dependent variables. To investigate whether patients and controls differed in overall symptom level, multilevel regression analyses were conducted using group as the independent variable, and using negative affect, positive affect, delusions and hallucinations as dependent variables in consecutive models.

\section{Self-medication effects}

Self-medication effects were examined using negative affect $_{\text {previous, }}$, positive affect $_{\text {previous }}$, delusions previous $_{\text {and hallucinations previous }}$ as independent variables, and cannabis use as the dependent variable (Fig. 1, analysis A).

\section{Cannabis effects in patients $v$. controls}

Main effects of cannabis use on subsequent symptom levels were investigated with cannabis use as independent, and negative affect, positive affect, delusions and hallucinations as dependent variables (Fig. 1, analysis B). In order to test the hypothesis that patients and controls differed in their sensitivity to cannabis effects, 


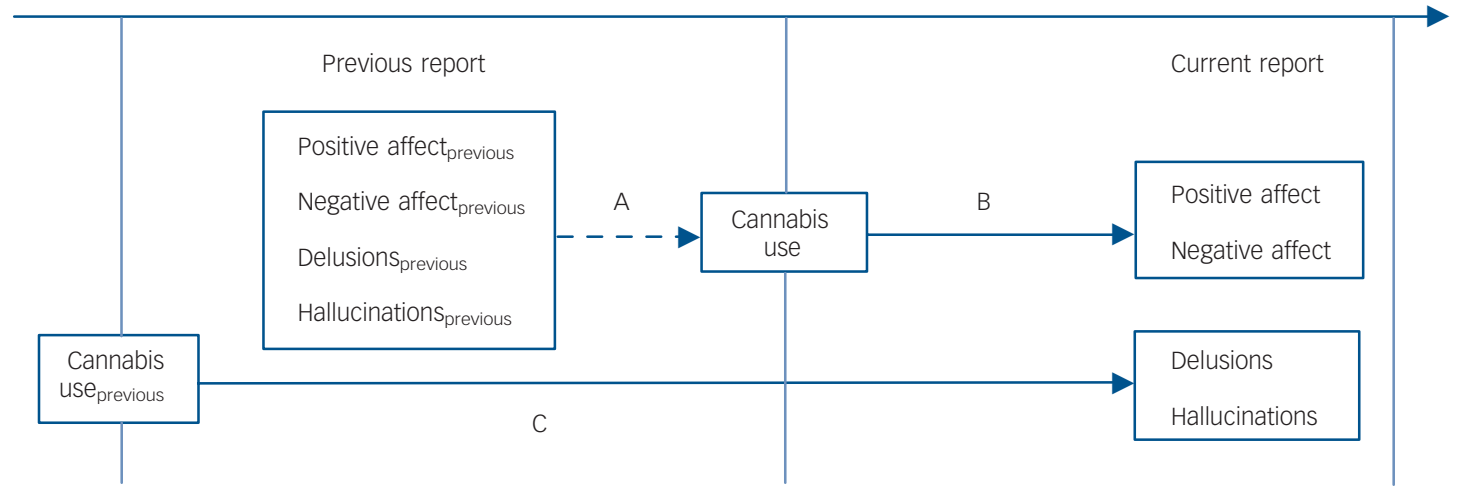

\section{Fig. 1 Experience sampling analysis}

A, analysis to investigate self-medication effects with negative affect $t_{\text {previous, }}$ positive affectprevious, delusions previous and hallucinations previous as independent variables and cannabis use as the dependent variable; B, analysis to investigate main effects of cannabis use with cannabis use as the independent variable and negative affect, positive affect, delusions and hallucinations as dependent variables; $C$, analyses (post-hoc in the patient group exclusively) to differentiate between sub-acute (C) and acute (B) effects of cannabis use on mood and positive symptoms.

multilevel regression analyses were conducted with cannabis use and group as well as their interaction term as independent variables and with negative affect, positive affect, delusions and hallucinations as dependent variables. Regression model: ESM symptom level $=\beta 0+\beta 1$ (cannabis use $)+\beta 2$ (group) $+\beta 3($ ESM cannabis use $\times$ group). This cannabis $\times$ group interaction was then fitted to allow estimation of cannabis effect sizes for both groups separately by calculating the appropriate linear combinations with the LINCOM command in STATA.

\section{Temporal dynamics of cannabis effects}

Based on the results of the previous analyses, post-hoc analyses were conducted to further investigate the duration of cannabis effects in the patient group only. To investigate these temporal dynamics, cannabis use (reported at the current beep) and

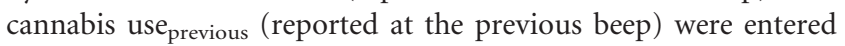
simultaneously in the same model predicting negative affect, positive affect and hallucinations (Fig. 1, analysis C).

All analyses were a priori adjusted for age, gender, alcohol use and overall level of cannabis use during the ESM week. The analyses investigating self-medication effects were additionally adjusted for cannabis use reported at the previous beep, given the fact that cannabis use was strongly associated with cannabis use $_{\text {previous }}(\mathrm{OR}=3.66,95 \%$ CI 3.05-4.40, $P<0.001)$. In order to account for possible self-medication effects (i.e. symptom level reported at the previous beep causing an increase in symptoms and cannabis use reported at the current beep) the analyses investigating main effects of cannabis use on symptom levels

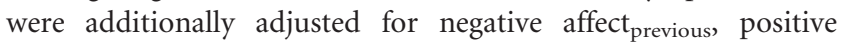
affect $_{\text {previous, delusions }}$ previous and hallucinations previous. Main effects and interaction were assessed by Wald test.

\section{Results}

\section{Participants}

Of the 95 participants included in the study, 9 controls and 6 patients had fewer than 24 valid reports and were excluded from the analyses. Drop-out was not associated with group $(\mathrm{OR}=0.94$, 95\% CI $0.32-2.73, P=0.91$ ) or cannabis use during the ESM week $(\beta=1.01,95 \%$ CI $0.88-1.16, P=0.90)$. The final study sample consisted of 80 participants (42 patients and 38 controls; Tables
1 and 2). The OPCRIT diagnoses according to RDC were: schizophrenia $(n=10)$, schizoaffective disorder $(n=28)$, and unspecified functional psychosis $(n=4)$. Groups differed significantly in age, but not in gender (Table 1), or in lifetime or current frequency of cannabis use or lifetime use of other drugs (Table 3).

\section{Overall symptom levels}

Overall, patients reported significantly lower levels of positive affect $(\beta=-0.56,95 \% \mathrm{CI}-1.03$ to $-0.09, P=0.019)$ and higher levels of negative affect than controls $(\beta=0.41,95 \%$ CI 0.07 to $0.75, P=0.020)$. Patients and controls differed significantly in the intensity of hallucinations they reported $(\beta=0.34,95 \% \mathrm{CI}$ 0.09 to $0.60, P=0.008)$, but not in levels of delusions $(\beta=0.26$, $95 \%$ CI -0.17 to $0.70, P=0.24)$. Frequency of cannabis use during the ESM week was significantly higher in patients than in controls $(\mathrm{OR}=1.76,95 \% \mathrm{CI} 1.12$ to $2.77, P=0.015)$.

\section{Self-medication effects}

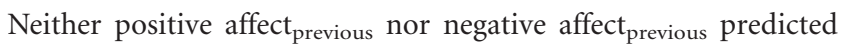
cannabis use reported at the following beep $(\mathrm{OR}=1.08,95 \% \mathrm{CI}$ $0.99-1.16, P=0.09$ and $\mathrm{OR}=0.97,95 \%$ CI $0.88-1.08, P=0.64$ respectively). Similarly, no association was found between delusions $_{\text {previous }}$ and subsequent cannabis use $(\mathrm{OR}=0.99,95 \%$ CI $0.890-1.06, P=0.97)$ or hallucinations previous $_{\text {and }}$ cannabis use $(\mathrm{OR}=0.96,95 \%$ CI $0.83-1.11, P=0.59)$.

\section{Cannabis effects on mood in patients $\boldsymbol{v}$. controls}

Cannabis use was associated with subsequent increases in positive affect ( $\beta=0.21,95 \%$ CI 0.14 to $0.29, P<0.001$ ). Overall, cannabis use had no effect on negative affect $(\beta=-0.04,95 \%$ CI -0.09 to $0.01, P=0.12$ ). The cannabis use $\times$ group interaction, however, was significant for negative affect. Thus, patients were more sensitive to the mood-enhancing effects of cannabis than controls (i.e. large and significant decreases in negative affect were observed after cannabis use in patients, but not in controls; cannabis $\times$ group interaction: $\chi^{2}=6.43$, d.f. $=1, P=0.011$; Table 4 ). No such interaction effect was observed for changes in positive affect after cannabis use $\left(\chi^{2}=0.98\right.$, d.f. $\left.=1, P=0.32\right)$. 
Table 1 Sociodemographics of the study sample

\begin{tabular}{|lcc|}
\hline & Controls $(n=38)$ & Patients $(n=42)$ \\
\hline Age, years: mean (s.d.) (range) & $26.9(7.5)(18-47)^{\mathrm{a}}$ & $36.1(9.3)(19-58)$ \\
\hline Gender (M/F) & $31 / 7^{\mathrm{b}}$ & $31 / 11$ \\
\hline Education, $n$ & & \\
Elementary school & 3 & 8 \\
Secondary school & 18 & 28 \\
Higher education & 17 & 3 \\
\hline Living situation, $n$ & & 22 \\
Alone & 19 & 9 \\
With partner/own family & 7 & 5 \\
With parents/other family & 12 & 6 \\
Protected housing & - & 4 \\
\hline Work situation, $n$ & & 8 \\
Working (e.g. school, household) & 27 & 26 \\
Unemployed & 7 & 1 \\
Incapable of work & 2 & \\
Protected work & - & \\
a. Age differed significantly between groups $(\beta=9.2,95 \%$ Cl 5.5-12.9, $P<0.001)$. \\
b. Gender did not differ significantly between groups $\left(\chi^{2}=0.691\right.$, d.f. $\left.=1, P=0.41\right)$. \\
\hline
\end{tabular}

\begin{tabular}{|c|c|c|}
\hline & Controls $(n=38)$ & Patients $(n=42)$ \\
\hline Total PANSS score, ${ }^{a}$ mean (s.d.) & $32.3(2.3)$ & $47.5(11.6)$ \\
\hline PANSS positive score, mean (s.d.) & $7.4(0.8)$ & $12.1(4.7)$ \\
\hline PANSS negative score, mean (s.d.) & $7.5(1.1)$ & $10.1(3.4)$ \\
\hline $\begin{array}{l}\text { Age first psychotic episode, years: } \\
\text { mean (s.d.) }\end{array}$ & - & $22.5(6.9)$ \\
\hline \multicolumn{3}{|l|}{$\begin{array}{l}\text { Usual symptom severity } \\
\text { (past } 2 \text { years), } n\end{array}$} \\
\hline Severe & - & 3 \\
\hline Mild to moderate & - & 19 \\
\hline Recovered & - & 4 \\
\hline \multicolumn{3}{|l|}{ Current use of medication, $n$} \\
\hline No antipsychotic medication & 38 & 7 \\
\hline Typical antipsychotics & - & 12 \\
\hline Atypical antipsychotics & - & 23 \\
\hline
\end{tabular}

\section{Cannabis effects on psychosis in patients $v$. controls}

Cannabis use was significantly associated with subsequent increases of hallucinations $(\beta=0.05,95 \%$ CI $0.01-0.08$, $P=0.015)$. No main effect of cannabis use was found for subsequent delusion intensity $(\beta=-0.02,95 \% \mathrm{CI}-0.07$ to $0.04, P=0.58$ ). Patients and controls differed in their sensitivity to the hallucinogenic effects of cannabis (cannabis $\times$ group interaction $\chi^{2}=3.66$, d.f. $=1, P=0.056$; Table 4). Patients reported significant increases in hallucinations $(\beta=0.08,95 \%$ CI $0.03-0.13$,
$P=0.002)$, whereas the association between cannabis use and hallucinations was small and non-significant in controls $(\beta=0.01$, $95 \%$ CI -0.04 to $0.06, P=0.75)$. Further post-hoc analyses for the separate hallucination items showed that the cannabis effects in patients were particularly associated with auditory hallucinations ('hearing voices', $\beta=0.11,95 \%$ CI $0.04-0.17, P=0.003$; Table 4) but that they were less clearly associated with visual hallucinations. No interaction effects were found for delusions $\left(\chi^{2}=1.17\right.$, d.f. $=1, P=0.28$; Table 4 ).

\section{Temporal dynamics of cannabis effects}

A follow-up post-hoc analysis was conducted, assessing the duration of cannabis effects on mood and hallucinations in the patient group only, by entering both cannabis use and cannabis use $_{\text {previous }}$ simultaneously in the same model. This suggested that increases in positive affect were observed in the short term ( $\beta=0.31,95 \%$ CI $0.19-0.43, P<0.001$ for cannabis use) rather than the long term $(\beta=0.07,95 \%$ CI -0.056 to $0.19, P=0.28$ for cannabis use previous $)$. For hallucinations, however, when both

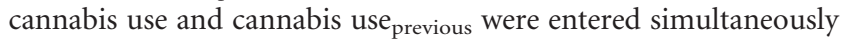
in the same model, increases in hallucinatory experiences were observed only in the long term $(\beta=0.09,95 \%$ CI $0.01-0.17$, $P=0.030$ for cannabis use $\left.\mathrm{p}_{\text {previous }}\right)$ and not in the short term ( $\beta=0.06,95 \%$ CI -0.01 to $0.14, P=0.11$ for cannabis use). This suggests that the association between cannabis and hallucinatory experiences was most prominent after a longer period of time compared with its shorter-term mood-enhancing effects.

\section{Discussion}

Cannabis use in daily life was associated with subsequent increases in hallucinatory experiences, in particular auditory hallucinations. Patients with a psychotic disorder were more sensitive to the hallucinogenic effects of cannabis than healthy controls. Overall, cannabis enhanced mood, with patients being more sensitive to the positive effects of cannabis on negative affect (i.e. stronger decreases in negative affect after cannabis use). In addition, the data suggest that the positive effects of cannabis on mood are acute, whereas its association with psychotic experiences is subacute. Neither negative affect nor hallucinations nor delusions predicted cannabis use, arguing against self-medication effects in daily life. In addition, the effect on subsequent symptom level remained both large and significant after adjustment of symptom level at the previous assessment (i.e. symptoms preceding the use of cannabis).

The current momentary assessment data confirm epidemiological and experimental findings, ${ }^{19,20}$ showing that patients with a psychotic disorder are more sensitive to the psychosis-inducing effects of cannabis than healthy controls. Furthermore, the current findings extend this to the real world of everyday life. The effect sizes were small but the cumulative effect may be considerable,

\begin{tabular}{|c|c|c|c|c|c|}
\hline & Controls $(n=38)$ & Patients $(n=42)$ & $\hat{a}^{a}$ & $95 \% \mathrm{Cl}$ & $P$ \\
\hline \multicolumn{6}{|l|}{ Cannabis use } \\
\hline Age first cannabis use, years: mean (s.d.) & $16.9(4.4)$ & $18.0(6.3)$ & -1.22 & -3.75 to 0.33 & 0.35 \\
\hline Use more than once daily (during most heavy use), $n$ & 30 & 37 & -0.24 & -0.90 to 0.41 & 0.46 \\
\hline Use more than once daily (during past 12 months), $n$ & 29 & 37 & 0.24 & -0.31 to 1.29 & 0.39 \\
\hline \multicolumn{6}{|l|}{ Cocaine and/or stimulants use, $n$} \\
\hline Lifetime use & 22 & 27 & 0.04 & -0.20 to 0.28 & 0.75 \\
\hline Use during past 12 months & 12 & 14 & 0.13 & -0.10 to 0.37 & 0.26 \\
\hline
\end{tabular}




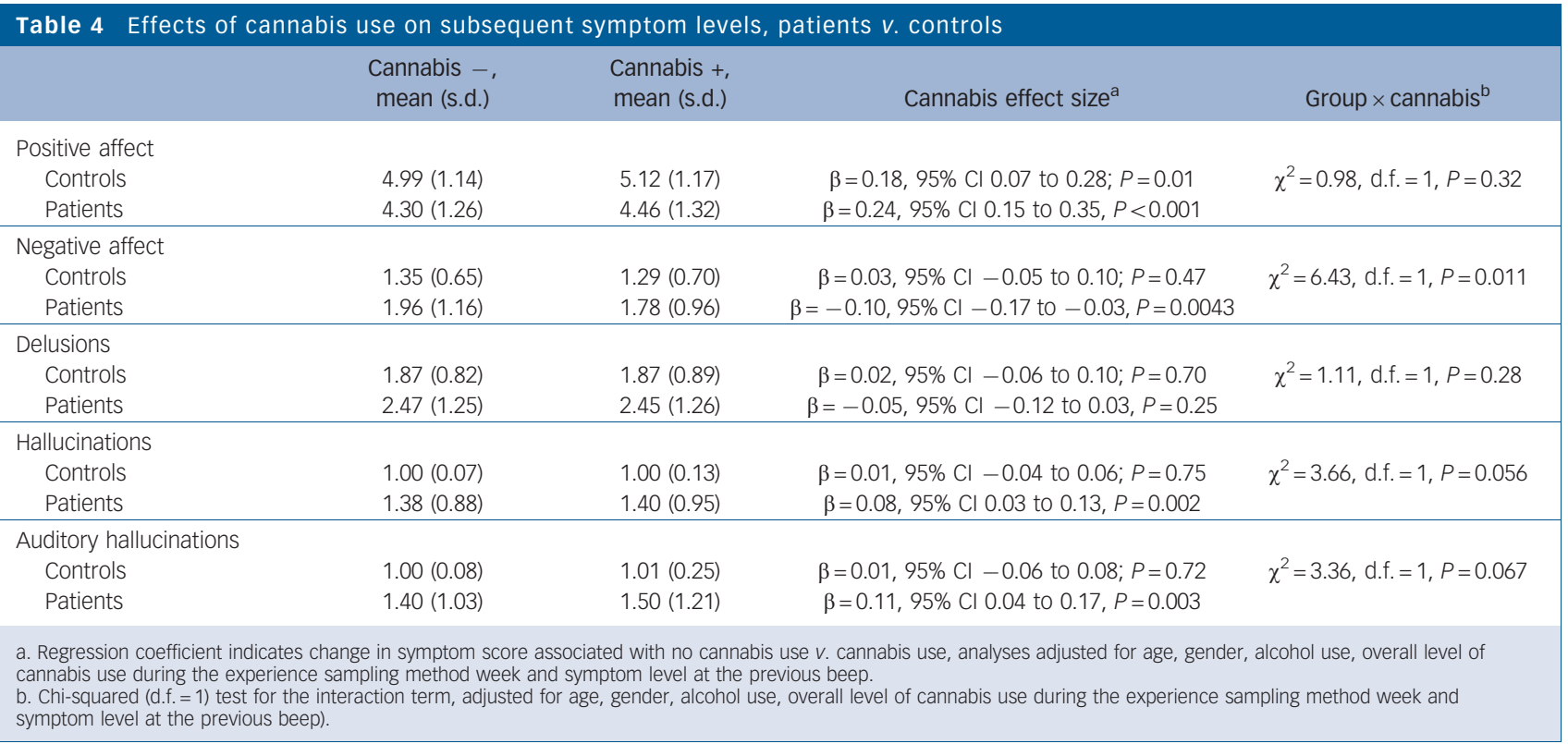

as exposure to cannabis and subsequent fluctuations in symptoms were observed several times a day over several days. The method of multiple assessments in daily life furthermore has the advantage of avoiding retrospective assessment of symptom states. These are most likely to be distorted by cannabis in the patient group, as increased sensitivity to cannabis operates not only at the symptom level, but also at the level of memory function. ${ }^{20} \mathrm{~A}$ crucial point in the interpretation of the results is the validity of the psychosis measures, especially because the results seem to indicate that hallucinations may be a more sensitive phenotype than delusions to study the acute effects of cannabis in daily life. Previous research has shown that patients can distinguish between hearing real voices and verbal hallucinations in self-report questionnaires, ${ }^{21}$ as well as between items such as preoccupation, suspicion and feeling as if controlled by others. ${ }^{15}$ In addition, the psychosis items used in this study are related to the same construct of psychosis, which is shown by a Cronbach's alpha of 0.71 . In a previous study, it was shown that these ESM hallucinations and delusions scores were significantly and specifically correlated with PANSS positive symptom scores $(r=0.45, P<0.001)$, but not with PANSS negative symptom scores $(r=0.26, P=0.12){ }^{22}$ Previous studies have also shown that ESM psychosis scores have discriminate validity, as they distinguish patients from first-degree relatives of patients with psychosis and healthy controls. ${ }^{22}$ The fact that in the current study patients did not differ in overall delusion levels from the control participants, may be explained by the long-term psychosis-inducing effects of chronic cannabis use which have been consistently reported in the general population as well. ${ }^{8,23}$ Comparison with a previous ESM study, indeed, showed that in the current controls using cannabis overall delusion levels were higher than in other control participants not using cannabis. ${ }^{24}$

\section{Increased addiction potential in patients}

In agreement with two longitudinal studies on this topic, ${ }^{8,9}$ the present data did not support direct mechanisms of selfmedication, since cannabis use was not predicted by previous changes in symptom level or mood. However, cannabis did improve mood, particularly in patients. The combination of differential sensitivity in patients to the acute rewarding effects and the sub-acute negative influences of cannabis on psychotic symptoms (despite the fact that the majority of patients were using antipsychotic medication) may be helpful in explaining the model of cannabis use in patients with psychosis, as proposed by Spencer et al. ${ }^{6}$ According to this model, use of cannabis is driven by expectations that individuals may have about the (acute) effects of cannabis. The sub-acute negative psychotic effects may then be experienced as evidence that more use is necessary to bring about the anticipated rewarding effects. Hallucinatory experiences are strongly associated with negative affect, ${ }^{18}$ fuelling further use in order to experience acute improvement in mood. Patients may be more sensitive to these mechanisms as they (a) have overall higher levels of negative affect and (b) are more sensitive to the mood- and psychosis-enhancing effects of cannabis. The motivation to enhance affect may consequently direct the individual to future use and cannabis dependence, despite the long-term negative impact cannabis may have on functional outcome. ${ }^{2,4}$ The finding that cannabis use was not predicted by changes in mood at the previous beep, could be explained by the fact that in the context of ongoing high levels of negative affect, patients may delay use until they find an appropriate time to smoke (with mood fluctuations occurring further back in the chain of events).

\section{Biological plausibility}

A reciprocal interaction between the endocannabinoid and dopamine system may explain the psychotogenic effects of cannabis in individuals with increased liability to psychosis such as the patient group. ${ }^{25}$ In the central nervous system, THC ( $\Delta$-9-tetrahydrocannabinol, the psychoactive component of cannabis) binds to cannabinoid 1 (CB1) receptors, the primary receptor site for endocannabinoids. Endocannabinoids act as retrograde signals at central nervous system synapses, by activating presynaptic CB1 receptors. ${ }^{26,27}$ Activation of $\mathrm{CB} 1$ receptors inhibits presynaptic neurotransmitter release. The endocannabinoid system thus plays an important role in the modulation of other neurotransmitters (e.g. gamma-aminobutyric acid and glutamate) and may thereby indirectly influence dopamine firing as well. ${ }^{28}$ Exogenous cannabinoids such as THC, however, disrupt these subtle, fine-tuning effects of endocannabinoids. ${ }^{29}$ Animal research has shown that THC evokes burst-firing in the ventral tegmental area and thereby increases dopamine concentrations in striatal 
brain regions. ${ }^{30,31}$ The cannabis effects as presented in this study might thus be attributable to the effects of THC on dopaminergic neurotransmission. Striatal dopamine is thought to play a crucial role in attributing salience to stimuli in the environment. As a consequence, a hyperdopaminergic state may facilitate psychotic experiences by enabling false attribution of significance to ambiguous stimuli. ${ }^{32}$ Auditory hallucinations may then be most prominent after exposure to THC as they occur in the moment, whereas delusions may be secondary interpretations of aberrant perceptions. ${ }^{33}$ Recently, the acute effects of THC on striatal dopamine were investigated for the first time in human healthy volunteers, using experimental positron emission tomography paradigms. Bossong et al found THC-induced striatal dopamine release, ${ }^{34}$ whereas Stokes and colleagues did not find such effects on dopamine transmission. ${ }^{35}$ These divergent findings may be due to the fact that individuals differ in their sensitivity to THC, with individuals expressing high psychosis liability, such as patients with schizophrenia, being more vulnerable than healthy controls. ${ }^{1,36}$ Epidemiological work has now identified several factors that co-participate with THC in causing psychosis, ${ }^{37}$ as pre-existing psychotic symptoms, ${ }^{8}$ exposure to childhood trauma $^{38,39}$ and a functional polymorphism in the catechol-Omethyltransferase gene ${ }^{40,41}$ have been shown to moderate the effects of cannabis on psychosis outcome. The results of the current study further support the idea that gene-environment interactions underlie the cannabis-psychosis association by showing that individuals at increased risk for psychosis (such as patients) are more sensitive to both the psychosis-inducing and mood-enhancing effects of THC.

\section{Limitations}

Several limitations need to be taken into account. First, reports on cannabis use and symptoms were based on self-report and were not confirmed by, for example, urine analysis. However, as consumption of cannabis is legal in The Netherlands and $32-51 \%$ of the assessments were cannabis moments, underreporting of drug use is unlikely. Experience sampling method adherence research ${ }^{42}$ has shown that individuals generally adhere very well with ESM procedures as carried out in the current investigation. Second, the validity of the reports may be challenged by negative effects of cannabis on cognition. The number of drop-outs due to invalid reports, however, was similar to that reported in a recent ESM adherence study. ${ }^{42}$ Third, patients were on average 10 years older than controls, thus duration of cannabis use may have been higher in patients than in controls. Adjustment for age did not change the results significantly. However, it may be useful to match on age and duration of cannabis use in future ESM studies. Fourth, it was found recently that patients with a first episode of psychosis show a preference for higher potency cannabis compared with healthy controls. ${ }^{43}$ In the current study, however, type and potency of cannabis was not controlled for in the analyses. It is unlikely, however, that this explains the stronger effects of cannabis on psychosis in the patient group. This is because the percentage of THC of the cannabis available in The Netherlands is correlated with its price and on average patients paid lower prices than controls for the cannabis they used during this study ( $\beta=-1.40,95 \% \mathrm{CI}-2.85$ to $0.05, P=0.058$ ). Nevertheless, in future ESM studies it would be interesting to include information on type and potency of cannabis (e.g. by means of hair samples, ${ }^{44}$ especially given the differential effects of THC and cannabidiol on mood and psychotic symptoms. ${ }^{45}$ Fifth, participants were regular cannabis users, which raises the question of whether the results would generalise to less frequent use of cannabis. D'Souza et $a l^{46}$ found frequent users to be blunted to the acute psychotomimetic effects of $\Delta$-9-THC. Epidemiological work however, has also shown that the long-term effects of cannabis may increase the risk for psychosis in a dose-response fashion, ${ }^{8}$ a finding that may be suggestive of a sensitisation process. More research is needed to investigate the moderating effect of duration of previous exposure on the acute psychosis-inducing effects of $\Delta-9$-THC.

\section{Clinical implications}

In patients, the clinical goal may be to intervene as early as possible to limit progression of the illness to more severe states associated with comorbidity of psychotic illness and cannabis misuse. Cannabis use is frequently a concern in treatment, yet it is often insufficiently discussed between patient and mental health provider. Since the consumption of cannabis and use-related activities play an important role in the everyday social lives of patients, it is of major importance to get better insight into the mechanisms and patterns of use. Some patients who were participating in this study reported that completion of the ESM booklets and the feedback they received afterwards on their patterns of use and related symptoms may have already changed their conceptions about cannabis use to some extent. This suggests that the data presented here may be of great clinical use, being applicable at the individual level as well. A combination of motivational interviewing and cognitive-behavioural therapy (in which not only the positive but also the affective symptoms are addressed) has been proposed to be most effective for patients with psychosis and comorbid substance misuse. ${ }^{47}$ The experience sampling data described here validate this idea by emphasising the need to recognise individual differences in sensitivity to cannabis and addiction potential resulting from differences in (genetic) liability to psychosis.

\footnotetext{
Cécile Henquet, PhD, Department of Psychiatry and Psychology, EURON, Maastricht University Medical Centre, South Limburg Mental Health Research and Teaching Network, Maastricht, and Mondriaan Zorggroep, Division Addiction Care, South Limburg, The Netherlands; Jim van Os, MD, PhD, Department of Psychiatry and Psychology, EURON, Maastricht University Medical Centre, South Limburg Mental Health Research and Teaching Network, Mastricht The Netherlands, and Division Psychological Medicine, Institute of Psychiatry, Kings College, London, UK; Rebecca Psychological Medicine, Institute of Psychiatry, Kings College, London, UK; Rebeccer
Kuepper, MSc, Department of Psychiatry and Psychology, EURON, Maastricht University Medical Centre, South Limburg Mental Health Research and Teaching Network, Maastricht, The Netherlands; Philippe Delespaul, PhD, Department of Psychiatry and Psychology, EURON, Maastricht University Medical Centre, South Limburg Mental Health Research and Teaching Network, Maastricht, and Mondriaan Zorggroep, South Limburg, The Netherlands; Maurice Smits, MSc, Joost à Campo, MD, PhD, Mondriaan Zorggroep, South Limburg, The Netherlands; Inez MyinGermeys, PhD, Department of Psychiatry and Psychology, EURON, Maastricht University Medical Centre, South Limburg Mental Health Research and Teaching Network, Maastricht, The Netherlands, and School of Psychological Sciences, University of Manchester, UK

Correspondence: Dr Cécile Henquet, Department of Psychiatry and Psychology, Maastricht University Medical Centre, PO BOX 616 (Loc. Vijverdal), 6200 MD Maastricht, The Netherlands. Email: cecile.henquet@sp.unimaas.nl

First received 19 Aug 2009, final revision 14 Jan 2010, accepted 28 Feb 2010
}

\section{Funding}

C.H. was supported by the Dutch Medical Research Council (VENI grant). M.S. was supported by the Dutch Medical Research Council (OOG grant). I.M.-G. was supported by the Dutch Medical Research Council (VIDI grant) and by a 2006 NARSAD Young Investigator Award.

\section{References}

1 Murray RM, Morrison PD, Henquet C, Di Forti M. Cannabis, the mind and society: the hash realities. Nat Rev Neurosci 2007; 8: 885-95.

2 Grech A, Van Os J, Jones PB, Lewis SW, Murray RM. Cannabis use and outcome of recent onset psychosis. Eur Psychiatry 2005; 20: 349-53. 
3 Caspari D. Cannabis and schizophrenia: results of a follow-up study. Eur Arch Psychiatry Clin Neurosci 1999; 249: 45-9.

4 Linszen DH, Dingemans PM, Lenior ME. Cannabis abuse and the course of recent-onset schizophrenic disorders. Arch Gen Psychiatry 1994; 51: 273-9.

5 Compton MT, Furman AC, Kaslow NJ. Lower negative symptom scores among cannabis-dependent patients with schizophrenia-spectrum disorders: preliminary evidence from an African American first-episode sample. Schizophr Res 2004; 71: 61-4.

6 Spencer C, Castle D, Michie PT. Motivations that maintain substance use among individuals with psychotic disorders. Schizophr Bull 2002; 28: 233-47.

7 Ferdinand RF, Sondeijker F, van der Ende J, Selten JP, Huizink A, Verhulst FC. Cannabis use predicts future psychotic symptoms, and vice versa. Addiction 2005; 100: 612-8.

8 Henquet C, Krabbendam L, Spauwen J, Kaplan C, Lieb R, Wittchen HU, et al. Prospective cohort study of cannabis use, predisposition for psychosis, and psychotic symptoms in young people. BMJ 2005; 330: 11-5.

9 Stefanis NC, Delespaul P, Henquet C, Bakoula C, Stefanis CN, Van Os J. Early adolescent cannabis exposure and positive and negative dimensions of psychosis. Addiction 2004; 99: 1333-41.

10 McGuffin P, Farmer A, Harvey I. A polydiagnostic application of operational criteria in studies of psychotic illness: development and reliability of the OPCRIT system. Arch Gen Psychiatry 1991; 48: 764-70.

11 Spitzer RL, Endicott J, Robins E. Research diagnostic criteria: rationale and reliability. Arch Gen Psychiatry 1978; 35: 773-82.

12 Kay SR, Fiszbein A, Opler LA. The positive and negative syndrome scale (PANSS) for schizophrenia. Schizophr Bull 1987; 13: 261-76.

13 Myin-Germeys I, van Os J, Schwartz JE, Stone AA, Delespaul PA. Emotional reactivity to daily life stress in psychosis. Arch Gen Psychiatry 2001; 58: s1137-44.

14 Myin-Germeys I, Krabbendam L, Jolles J, Delespaul PA, van Os J. Are cognitive impairments associated with sensitivity to stress in schizophrenia? An experience sampling study. Am J Psychiatry 2002; 159: 443-9.

15 Delespaul PAEG. Empirical studies in ESM reliability and validity. In Assessing Schizophrenia in Daily Life: The Experience Sampling Method. (ed. MW de Vries): 107. Maastricht University Press, 1995.

16 Myin-Germeys I, Marcelis M, Krabbendam L, Delespaul P, van Os J. Subtle fluctuations in psychotic phenomena as functional states of abnormal dopamine reactivity in individuals at risk. Biol Psychiatry 2005; 58: 105-10.

17 Van Winkel R, Henquet C, Rosa A, Papiol S, Fananas L, De Hert M, et al. Evidence that the COMT(Val158Met) polymorphism moderates sensitivity to stress in psychosis: an experience-sampling study. Am J Med Genet B Neuropsychiatr Genet 2008; 147: 10-7.

18 Delespaul $\mathrm{P}$, deVries $\mathrm{M}$, van Os J. Determinants of occurrence and recovery from hallucinations in daily life. Soc Psychiatry Psychiatr Epidemiol 2002; 37 97-104.

19 Van Os J, Bak M, Hanssen M, Bijl RV, de Graaf R, Verdoux H. Cannabis use and psychosis: a longitudinal population-based study. Am J Epidemiol 2002; 156: 319-27.

20 D'Souza DC, Abi-Saab WM, Madonick S, Forselius-Bielen K, Doersch A, Braley G, et al. Delta-9-tetrahydrocannabinol effects in schizophrenia: implications for cognition, psychosis, and addiction. Biol Psychiatry 2005; 57: 594-608.

21 Romme MA, Honig A, Noorthoorn EO, Escher AD. Coping with hearing voices: an emancipatory approach. Br J Psychiatry 1992; 161: 99-103.

22 Myin-Germeys I, Delespaul P, van Os J. Behavioural sensitization to daily life stress in psychosis. Psychol Med 2005; 35: 733-41.

23 Arseneault L, Cannon M, Poulton R, Murray R, Caspi A, Moffitt TE. Cannabis use in adolescence and risk for adult psychosis: Iongitudinal prospective study. BMJ 2002; 325: 1212-3.

24 Thewissen V, Bentall RP, Lecomte T, van Os J, Myin-Germeys I. Fluctuations in self-esteem and paranoia in the context of daily life. J Abnorm Psychol 2008; 117: 143-53.

25 Giuffrida A, Leweke FM, Gerth CW, Schreiber D, Koethe D, Faulhaber J, et al. Cerebrospinal anandamide levels are elevated in acute schizophrenia and are inversely correlated with psychotic symptoms. Neuropsychopharmacology 2004; 29: 2108-14
26 Wilson RI, Nicoll RA. Endogenous cannabinoids mediate retrograde signalling at hippocampal synapses. Nature 2001; 410: 588-92.

27 Elphick MR, Egertova M. The neurobiology and evolution of cannabinoid signalling. Philos Trans R Soc Lond B Biol Sci 2001; 356: 381-408.

28 Chevaleyre V, Takahashi KA, Castillo PE. Endocannabinoid-mediated synaptic plasticity in the CNS. Ann Rev Neurosci 2006; 29: 37-76.

29 Leweke FM, Koethe D. Cannabis and psychiatric disorders: it is not only addiction. Addict Biol 2008; 13: 264-75.

30 Cheer JF, Wassum KM, Heien ML, Phillips PE, Wightman RM. Cannabinoids enhance subsecond dopamine release in the nucleus accumbens of awake rats. J Neurosci 2004; 24: 4393-400.

31 Riegel AC, Lupica CR. Independent presynaptic and postsynaptic mechanisms regulate endocannabinoid signaling at multiple synapses in the ventral tegmental area. J Neurosci 2004; 24: 11070-8.

32 Kapur S, Mizrahi R, Li M. From dopamine to salience to psychosis: linking biology, pharmacology and phenomenology of psychosis. Schizophr Res 2005; 79: 59-68.

33 Bentall RP, Kinderman P, Kaney S. The self, attributional processes and abnormal beliefs: towards a model of persecutory delusions. Behav Res Ther 1994; 32: 331-41.

34 Bossong MG, van Berckel BN, Boellaard R, Zuurman L, Schuit RC, Windhorst $A D$, et al. Delta 9-tetrahydrocannabinol induces dopamine release in the human striatum. Neuropsychopharmacology 2009; 34: 759-66.

35 Stokes PR, Mehta MA, Curran HV, Breen G, Grasby PM. Can recreational doses of THC produce significant dopamine release in the human striatum? Neurolmage 2009; 48: 186-90.

36 Henquet C, Di Forti M, Morrison P, Kuepper R, Murray RM. Gene-environment interplay between cannabis and psychosis. Schizophr Bull 2008; 34: 1111-21.

37 Arseneault L, Cannon M, Witton J, Murray RM. Causal association between cannabis and psychosis: examination of the evidence. Br J Psychiatry 2004; 184: $110-7$.

38 Houston JE, Murphy J, Adamson G, Stringer M, Shevlin M. Childhood sexual abuse, early cannabis use, and psychosis: testing an interaction model based on the National Comorbidity Survey. Schizophr Bull 2008; 34: 580-5.

39 Harley M, Kelleher I, Clarke M, Lynch F, Arseneault L, Connor D, et al. Cannabis use and childhood trauma interact additively to increase the risk of psychotic symptoms in adolescence. Psychol Med 2009; 9: 1-8.

40 Caspi A, Moffitt TE, Cannon M, McClay J, Murray R, Harrington H, et al. Moderation of the effect of adolescent-onset cannabis use on adult psychosis by a functional polymorphism in the catechol-O-methyltransferase gene: longitudinal evidence of a gene $\times$ environment interaction. Biol Psychiatry 2005; 57: 1117-27.

41 Henquet C, Rosa A, Krabbendam L, Papiol S, Fananas L, Drukker M, et al. An experimental study of catechol-O-methyltransferase Val(158)Met moderation of delta-9-tetrahydrocannabinol-induced effects on psychosis and cognition. Neuropsychopharmacology 2006; 31: 2748-57.

42 Jacobs N, Nicolson NA, Derom C, Delespaul P, van Os J, Myin-Germeys I. Electronic monitoring of salivary cortisol sampling compliance in daily life. Life Sci 2005; 76: 2431-43.

43 Di Forti M, Morgan C, Dazzan P, Pariante C, Mondelli V, Marques TR, et al High-potency cannabis and the risk of psychosis. Br J Psychiatry 2009; 195: 488-91.

44 Morgan CJA, Curran HV. Effects of cannabidiol on schizophrenia-like symptoms in people who use cannabis. Br J Psychiatry 2008; 192: 306-7.

45 Bhattacharyya S, Morrison PD, Fusar-Poli P, Martin-Santos R, Borgwardt S, Winton- Brown T, et al. Opposite effects of delta-9-tetrahydrocannabinol and cannabidiol on human brain function and psychopathology. Neuropsychopharmacology 2010; 35: 764-74.

46 D'Souza DC, Ranganathan M, Braley G, Gueorguieva R, Zimolo Z, Cooper T, et al. Blunted psychotomimetic and amnestic effects of delta-9-tetrahydrocannabinol in frequent users of cannabis. Neuropsychopharmacology 2008; 33: 2505-16.

47 Barrowclough C, Haddock G, Tarrier N, Lewis SW, Moring J, O'Brien R, et al. Randomized controlled trial of motivational interviewing, cognitive behavior therapy, and family intervention for patients with comorbid schizophrenia and substance use disorders. Am J Psychiatry 2001; 158: 1706-13. 